\title{
Identification of the TW Hydrae association member 2M1235-39: a tertiary component of the HR 4796 system (Research Note)
}

\author{
J. H. Kastner ${ }^{1,2}$, B. Zuckerman ${ }^{3,4}$, and M. Bessell ${ }^{5}$ \\ ${ }^{1}$ Laboratoire d'Astrophysique de Grenoble, Université Joseph Fourier - CNRS, BP 53, 38041 Grenoble Cedex, France \\ e-mail: joel.kastner@obs.ujf-grenoble.fr \\ 2 Chester F. Carlson Center for Imaging Science, Rochester Institute of Technology, 54 Lomb Memorial Dr., Rochester, \\ NY 14623, USA \\ 3 Dept. of Physics \& Astronomy, University of California, Los Angeles 90095, USA \\ 4 UCLA Center for Astrobiology, University of California, Los Angeles 90095, USA \\ 5 Research School of Astronomy and Astrophysics, The Australian National University, Weston, ACT 2611, Australia
}

Received 12 July 2008 / Accepted 7 August 2008

\section{ABSTRACT}

\begin{abstract}
Aims. We seek to determine whether the late-type star 2MASS J12354893-3950245 (2M1235-39) is a member of the TW Hya Association (TWA), a hypothesis suggested by its association with a bright X-ray source detected serendipitously by ROSAT and XMM-Newton and its $\left(\sim 3^{\prime}\right)$ proximity to the well-studied (A+M binary) system HR 4796.

Methods. We used optical spectroscopy to establish the $\mathrm{Li}$ and $\mathrm{H} \alpha$ line strengths of 2M1235-39, and determined its proper motion via optical imaging. We also considered its X-ray and near-IR fluxes relative to the M star HR 4796B.

Results. The optical spectrum of 2M1235-39 displays strong Li absorption and H $\alpha$ emission (equivalent widths of $630 \mathrm{~m} \AA$ and $-6.7 \AA$, respectively). Comparison of the spectrum with that of a nearby field star, along with the DENIS catalog IJK magnitudes, indicates the spectral type of 2M1235-39 is M4.5. We measure a proper motion for 2M1235-39 that agrees, within the errors, with that of HR 4796.

Conclusions. The Li absorption and $\mathrm{H} \alpha$ emission line strengths of 2M1235-39, its near-IR and X-ray fluxes, and its proper motion all indicate that 2M1235-39 is a TWA member. Most likely this star is a wide (13500 AU) separation, low-mass, tertiary component of the HR 4796 system.
\end{abstract}

Key words. stars: individual: 2M1235-39 - stars: pre-main sequence - stars: individual: HR 4796

\section{Introduction}

As of little more than a decade ago, astronomers were almost oblivious to the presence of low-mass, pre-main sequence stars within $\sim 100 \mathrm{pc}$ of Earth. The intervening years have seen the identification of a few hundred such stars, with ages ranging from 8 to $100 \mathrm{Myr}$, as part of numerous post-T Tauri associations (Zuckerman \& Song 2004, hereafter ZS04, and references therein; Torres et al. 2006, 2008). Perhaps the greatest excitement associated with the recognition of the existence of nearby young stars has been the opportunity to study, at close range, the evolution of youthful planetary systems, via direct thermal imaging of warm massive planets (e.g. Chauvin et al. 2004; Song et al. 2006) and via imaging and spectroscopy of debris disks (e.g. Rebull et al. 2008, and references therein). Young, local stellar groups also afford unique insight into the early evolution of low-mass stars and ultracool dwarfs (e.g. Looper et al. 2007; Cruz et al. 2008, and references therein).

The difficulty inherent in identifying young stars and young star groups near Earth reflects the fact that such groups are spread over large areas of the sky (ZS04). Furthermore, while the local young groups are usually "spearheaded" by a handful of well-studied, individual systems that feature, e.g. strong $\mathrm{H} \alpha$ emission, enormous IR excesses, and/or easily imaged debris disks (TW Hya and $\beta$ Pic being cases in point), the vast majority of nearby young stars are otherwise unremarkable latetype (K through $\mathrm{M}$ ) dwarfs that do not stand out or even turn up in optical emission-line or far-infrared (e.g. IRAS) surveys.

However, all 10-100 Myr-old stars of types F through M are at or near the peaks of their lives in terms of their X-ray luminosities relative to bolometric (with "saturated" values of $L_{\mathrm{X}} / L_{\text {bol }} \sim 10^{-3}$, ZS04 (their Fig. 4); see also Kastner et al. 1997 and Preibisch \& Feigelson 2005). Hence, X-ray point source catalogs, in tandem with recently released, comprehensive catalogs of distances and proper motions of stars in the solar neighborhood, have served as the main resources with which to isolate stars that are likely nearby and young. Followup optical spectroscopy and/or imaging then readily confirms (or refutes) membership in the "nearby young star club", via determination of surface Li abundances and relative $(U V W)$ Galactic space motions.

Here, we demonstrate that serendipitous XMM-Newton and ROSAT X-ray detections of 2MASS J12354893-3950245 (hereafter 2M1235-39), combined with its optical spectrum and proper motion, establishes this star as a member of the quintessential local young star group, the TW Hya Association (TWA; Kastner et al. 1997; Webb et al. 1999; Zuckerman et al. 2001). Indeed, 2M1235-39 is, likely, the tertiary component of 
the well-studied HR 4796 (A+M star) binary system (Jura et al. 1993; Stauffer et al. 1995), which is designated TWA 11.

\section{Observations and results}

\subsection{Serendipitous $X$-ray detections of $2 M 1235-39$}

\subsubsection{ROSAT}

No X-ray sources are associated with 2M1235-39 in the ROSAT All-Sky Survey Bright or Faint Source Catalogs. However, the HR 4796 system was the subject of a pointed $41 \mathrm{ks}$ ROSAT HRI observation (Jura et al. 1998). That observation resulted in detections of HR $4796 \mathrm{~B}$ and a source within $5^{\prime \prime}$ of the position of 2M1235-39 (ROSAT HRI count rates ${ }^{1} 23.7 \pm 0.8 \mathrm{ks}^{-1}$ and $8.4 \pm 0.5 \mathrm{ks}^{-1}$, respectively). The ROSAT/HRI detection of HR 4796B was used by Jura et al. to establish that the X-ray source was centered on this $\mathrm{M}$ star, rather than on the primary (A-type) star. The detection of 2M1235-39 was not noted by these authors, however ${ }^{2}$.

\subsubsection{XMM-Newton}

The serendipitous XMM-Newton detection of 2M1235-39 is summarized in Lopez-Santiago et al. (2007), who analyzed 58 bright (EPIC-MOS2 $0.5-4.5 \mathrm{keV}$ count rate $>10 \mathrm{k} \mathrm{s}^{-1}$ ) X-ray sources with stellar counterparts that were detected in the XMM Bright Serendiptious Survey (XBSS). In the Lopez-Santiago et al. study, 2M1235-39 was identified as a star of M4 spectral type, where this spectral type is an estimate based its 2MASS colors. A better defined color-index for spectral typing of midM-type stars is $\mathrm{I}-\mathrm{K}$ which, from DENIS, indicates a spectral type of M4.5.

Given the relative paucity of serendipitous XMM sources as bright as that associated with 2M1235-39 (Lopez-Santiago et al. 2007), the proximity of the 2M1235-39 X-ray source to HR 4796AB (angular displacement of $\sim 3^{\prime}$ ) as well as the similarity of the X-ray fluxes and temperatures determined for the 2M1235-39 and HR 4796B sources by Lopez-Santiago et al. (2007) suggested to us that 2M1235-39 might be an as-yet unrecognized member of the TWA and, perhaps, a widely separated companion to $\mathrm{HR} 4796 \mathrm{AB}$.

\subsection{Optical spectroscopy of $2 M 1235-39$ and reference star}

To test the foregoing hypothesis, we obtained moderateresolution $(R \sim 1.16 \AA)$ red (6500-7500 $\AA$ region) spectra of 2M1235-39 and the nearby, M4 spectral type field star LHS 337 with the Double Beam Spectrograph (DBS) grating spectrometer on the $2.3 \mathrm{~m}$ Siding Spring Observatory (SSO) telescope. Results for the spectral range covering the $\lambda 6563 \mathrm{H} \alpha$ and $\lambda 6708 \mathrm{Li}$ I lines are displayed in Fig. 1. As expected for a member of the TWA, 2M1235-39 displays strong Li absorption and $\mathrm{H} \alpha$ emission, with respective equivalent widths $630 \mathrm{~m} \AA$ and $-6.66 \AA$. The lithium measurement confirms the youth of this star, as $\mathrm{M}$ dwarfs do not retain measureable abundances of Li beyond 230 Myr (see ZS04 and references therein). Furthermore, the spectral similarity of 2M1235-39 to LHS 337 (apart from the

\footnotetext{
1 The HRI X-ray count rates listed here were obtained from the HEASARC ROSAT archive; the count rate for HR 4796B is consistent with that determined by Jura et al. (1998).

2 Indeed, the recognition that HR 4796 is a member of the TWA (Webb et al. 1999) came subsequent to publication of Jura et al. (1998).
}
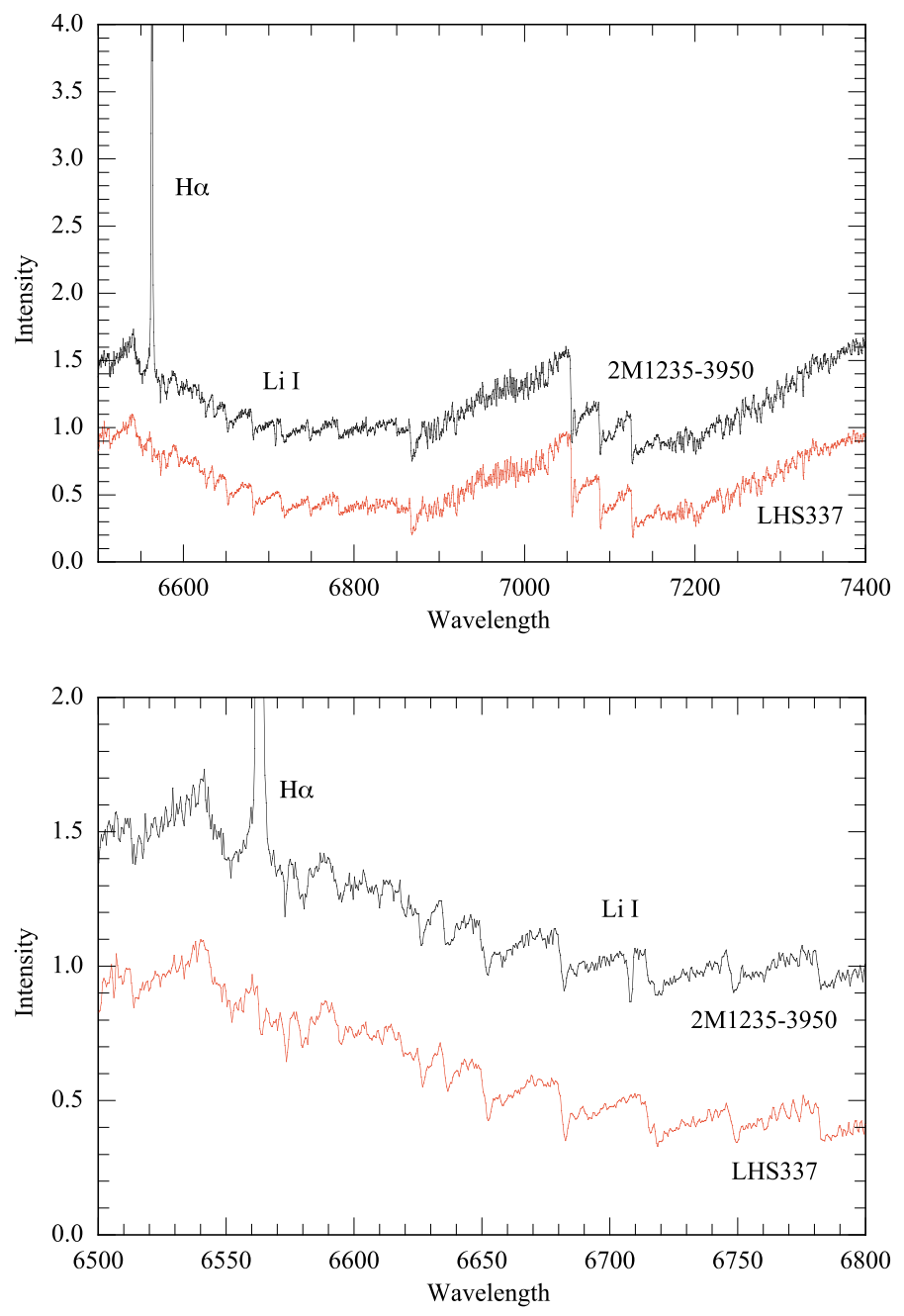

Fig. 1. Spectra of 2M1235-39 and a field star (LHS 337) of M4 spectral type, obtained with the Double Beam Spectrograph on the $2.3 \mathrm{~m}$ Siding Spring Observatory telescope (top: entire spectral range, bottom: closeup of the $\mathrm{H} \alpha$ and $\mathrm{Li}$ spectral region). Note the deep Li absorption and strong $\mathrm{H} \alpha$ emission in the 2M1235-39 spectrum. These spectral features are typical of the $8 \mathrm{Myr}-$ old stars of the TWA.

$\mathrm{Li}$ and $\mathrm{H} \alpha$ lines) confirms that its spectral subtype is consistent with M4.5.

\subsection{Proper motion of $2 M 1235-39$}

To ascertain the proper motion of 2M1235-39, we obtained a set of $I$ band images of the field with a $512 \times 512$ CCD camera on the $1 \mathrm{~m}$ telescope of the University of Tasmania Mt. Canopus Observatory on 10 June 2008 (epoch 2008.44). The camera pixel scale is $0.434 \pm 0.003 \operatorname{arcsec~pix}^{-1}$. With these images and the ESO 1982.37 epoch archival digitized sky image of the 2M1235-39 field, the proper motion of 2M1235-39 was measured using the IRAF task geomap (Rodriguez 2008, personal comm.). The resulting proper motion is $-49.6 \pm 3$ and $-25.1 \pm 3 \mathrm{mas} \mathrm{yr}^{-1}$ in RA and Dec, respectively. Within the respective errors, these proper motions agree with an average of those measured for HR 4796A in the Hipparchos, TYCHO, and PPM catalogs, i.e., $(-55.9,-24),(-53.3,-21.2)$, and $(-46$, $-18)$ mas $\mathrm{yr}^{-1}$, respectively. 


\section{Discussion}

The strong Li absorption measured for 2M1235-39 is compatible with TWA membership (e.g. Kastner et al. 1997; Webb et al. 1999; Zuckerman et al. 2001; Song et al. 2003). Specifically, the previously established member stars of the TWA that are of M spectral type have $6708 \AA \mathrm{Li}$ absorption line EWs in the range 360-650 m $\AA$. In addition, its $\mathrm{H} \alpha$ emission-line strength is near the median for M stars in the TWA.

Adopting a conversion factor of $1.1 \times 10^{-11} \mathrm{erg} \mathrm{cm}^{-2}$ count $^{-1}$ (Jura et al. 1998), the respective ROSAT/HRI (0.1-2.4 keV) $\mathrm{X}$-ray fluxes are $9.2 \times 10^{-14} \mathrm{erg} \mathrm{cm}^{-2} \mathrm{~s}^{-1}$ and $2.8 \times$ $10^{-13} \mathrm{erg} \mathrm{cm}^{-2} \mathrm{~s}^{-1}$ for the 2M1235-39 and HR 4796B X-ray sources, respectively, whereas Lopez-Santiago et al. (2007) derive respective intrinsic $(0.5-10 \mathrm{keV}) \mathrm{X}$-ray fluxes of $F_{\mathrm{X}}=$ $2.2 \times 10^{-13} \mathrm{erg} \mathrm{cm}^{-2} \mathrm{~s}^{-1}$ and $F_{\mathrm{X}}=1.4 \times 10^{-12} \mathrm{erg} \mathrm{cm}^{-2} \mathrm{~s}^{-1}$ from spectral modeling of the XMM-Newton EPIC CCD data. As the X-ray spectra of both sources display essentially no absorption (Lopez-Santiago et al. 2007), the latter intrinsic fluxes also well represent their observed $0.5-10 \mathrm{keV}$ fluxes as measured by XMM. The discrepancies between the fluxes of the 2M1235-39 and HR 4796B X-ray sources as measured by XMM/EPIC vs. ROSAT/HRI - i.e., a factor $\sim 2.5$ for $2 \mathrm{M} 1235-39$ and $\sim 5$ for HR 4796B - are likely due in large part to the superior hard $\mathrm{X}$-ray sensitivity of XMM/EPIC, since each source displays not only a soft $(k T \sim 0.3 \mathrm{keV})$ but a hard $(k T \sim 1.0 \mathrm{keV}) \mathrm{X}$-ray component in XMM/EPIC spectra (Lopez-Santiago et al.). It is also likely that these sources display X-ray source variability typical of pre-MS stars. In any event, the flux of the X-ray source associated with 2M1235-39 is compatible with TWA membership, given its mid-M spectral type (e.g. Kastner et al. 1997).

The proper motion of 2M1235-39 is also consistent with TWA membership and, as noted in Sect. 2.3, agrees within the errors with that of the HR 4796AB (=TWA 11AB = CD-39 $7717 \mathrm{AB}^{3}$ ) binary system. If $2 \mathrm{M} 1235-39$ is at the revised Hipparcos-measured distance of HR 4796A, $72.8 \mathrm{pc}$ (van Leeuwen 2007), then the absolute $K$ magnitude of 2M1235-39 is 4.5, based on the 2MASS and DENIS catalogs. This $M_{\mathrm{K}}$ places 2 M1235-39 within or perhaps slightly above the locus of 5-8 Myr-old stars in the TWA and the $\eta$ Cha cluster, very near the position of a binary M4.5 system in $\eta$ Cha (e.g. Fig. 2 in Song et al. 2003 and Fig. 2 in ZS04). Its similarity to the latter system suggests that 2M1235-39 may be a close binary. Given these considerations and the very low areal density of known TWA members, most likely 2M1235-39 ("TWA 11C") and TWA $11 \mathrm{AB}$ are physically bound, with a projected separation of $13500 \mathrm{AU}$. This would make TWA 11AB and TWA 11C the widest known binary in the TWA by a large margin (the previously identified TWA binary systems all have separations less than $\sim 15^{\prime \prime}$, or $\left.\sim 750 \mathrm{AU}\right)$.

\section{Conclusions}

All measured properties of 2M1235-39 presented here - its $\mathrm{Li}$ absorption and $\mathrm{H} \alpha$ emission line strengths, its near-IR and X-ray fluxes, and its proper motion - are compatible with TWA membership. Based on these results and on the similarity of its common proper motion to that of HR 4796A, we conclude that 2M1235-39 is most likely a wide (13500 AU) separation, lowmass, tertiary component of the HR 4796 system.

Acknowledgements. The authors are grateful to John Greenhill for obtaining the set of $I$ band images and David Rodriguez and Jay Farihi for analyzing these current epoch images of 2M1235-39. We thank Thierry Forveille for alerting us to the revised Hipparcos distance to HR 4796. J.H.K. thanks the staff of the Laboratoire d'Astrophysique de Grenoble for their support and hospitality during his yearlong sabbatical visit to that institution. This research was partially supported by a NASA grant to UCLA.

\section{References}

Chauvin, G., Lagrange, A.-M., Dumas, C., et al. 2004, A\&A, 425, L29 Cruz, K., Kirkpatrick, J. D., Burgasser, A., et al. 2008, in 14th Workshop on Cool Stars, Stellar Systems, and the Sun, ed. G. van Belle, ASP Conf. Ser., 384, 119

Jura, M., Zuckerman, B., Becklin, E. E., \& Smith, R. C. 1993, ApJ, 418, L37 Jura, M., Malkan, M., White, R., et al. 1998, ApJ, 505, 897

Kastner, J. H., Zuckerman, B., Forveille, T., \& Weintraub, D. A. 1997, Science, 277, 67

Looper, D. L., Burgasser, A. J., Kirkpatrick, J. D., \& Swift, B. J. 2007, ApJ, 669, L97

Lopez-Santiago, J., Micela, G., Sciortino, S., et al. 2007, A\&A, 463, 165

Preibisch, T., \& Feigelson, E. 2005, ApJS, 160, 390

Rebull, L., Stapelfeldt, K. R., Werner, M. W., et al. 2008, ApJ, in press [arXiv: 0803.1674]

Song, I., Zuckerman, B., \& Bessell, M. S. 2003, ApJ, 599, 342

Song, I., Schneider, G., Zuckerman, B., et al. 2006, ApJ, 652, 724

Stauffer, J., Hartmann, L. W., \& Barrado y Navascues, D. 1995, ApJ, 454, 910

Turner, T. J., George, I. M., Nandra, K., \& Mushotzky, R. F. 1997, ApJS, 113, 23

Torres, C. A. O., Quast, G. R., da Silva, L., et al. 2006, A\&A, 460, 695

Torres, C. A. O., Quast, G. R., Melo, C. H. F., \& Sterzik, M. F. 2008, in Handbook of Star Forming Regions, ed. B. Reipurth (San Francisco: ASP), in prep.

van Leeuwen, F. 2007, A\&A, 474, 653

Webb, R., Zuckerman, B., Platais, I., et al. 1999, ApJ, 512, L63

Zuckerman, B., \& Song, I. 2004, ARA\&A, 42, 685 (ZS04)

Zuckerman, B., Webb, R. A., Schwartz, M., \& Becklin, E. E. 2001, ApJ, 549, L233

\footnotetext{
3 The identification of an ASCA X-ray field source as "CD-39 7717C" in Turner et al. (1997) appears to be spurious. This source is, most likely, X-ray emission from HR 4796B or (perhaps) a combination of flux from HR 4796B and 2M1235-39, since the (poorly-determined) ASCA position for this source - while closer to HR 4796B - does not correspond well to the position of either star.
} 